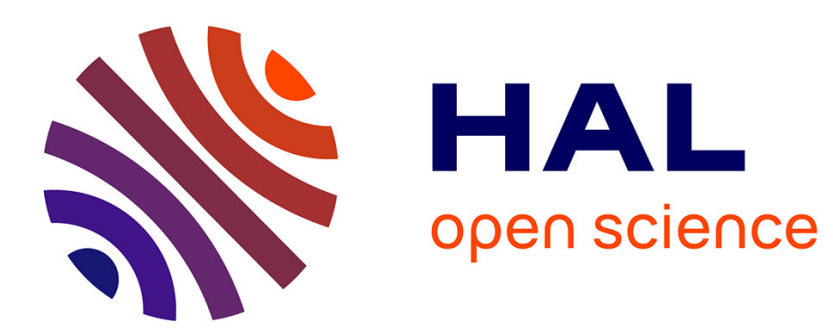

\title{
Rigid polymer network models
}

\author{
J.L. Jones, C.M. Marques
}

\section{To cite this version:}

J.L. Jones, C.M. Marques. Rigid polymer network models. Journal de Physique, 1990, 51 (11), pp.1113-1127. 10.1051/jphys:0199000510110111300 . jpa-00212434

\section{HAL Id: jpa-00212434 https://hal.science/jpa-00212434}

Submitted on 1 Jan 1990

HAL is a multi-disciplinary open access archive for the deposit and dissemination of scientific research documents, whether they are published or not. The documents may come from teaching and research institutions in France or abroad, or from public or private research centers.
L'archive ouverte pluridisciplinaire HAL, est destinée au dépôt et à la diffusion de documents scientifiques de niveau recherche, publiés ou non, émanant des établissements d'enseignement et de recherche français ou étrangers, des laboratoires publics ou privés. 
Classification

Physics Abstracts

$62.20 \mathrm{D}-81.40-03.40 \mathrm{D}$

\title{
Rigid polymer network models
}

\author{
J. L. Jones ( $\left.{ }^{1}\right)$ and C. M. Marques $\left({ }^{2, *}\right)$ \\ (1) Cavendish Laboratory, Cambridge CB3 OHE U.K. \\ (2) Ecole Normale Supérieure de Lyon, 69364 Lyon Cedex 07, France
}

(Reçu le 6 novembre 1989, accepté sous forme définitive le 20 février 1990)

\begin{abstract}
Résumé. - Nous présentons un modèle décrivant l'élasticité d'un réseau de chaînes rigides. La variation du module élastique avec la fraction volumique de polymère est déterminée dans les deux cas où : (a) les chaînes sont rigidement connectées entre elles, c'est-à-dire que les seules déformations possibles du réseau sont celles obtenues en courbant les chaines (réseau énergétique) et (b) les chaînes peuvent s'articuler librement autour des points d'interconnection et une entropie est alors associée à ces degrés de liberté. Nous définissons une concentration $\phi_{\mathrm{c}}$ de raccordement entre ces deux comportements. Un renforcement du réseau rigide peut être effectué en introduisant une petite concentration $c_{P}$ de bâtonnets de taille $P$ (beaucoup plus grande que la distance moyenne $L$ entre points de réticulation. Le module élastique se voit dans ces conditions augmenté d'un facteur, $G_{P} \propto c_{P} P$. Nous discutons en conclusion le gonflement et le "dégonflement" des réseaux rigides par le changement de la qualité du solvant. Nous calculons une forme possible du taux de gonflement en fonction de la taille de la maille du réseau. Le système est instable pour un taux de gonflement supérieur à $v_{2 \text { eq }}=(5 / 4)^{3}$.

Abstract. - A model is presented for the elasticity of networks composed of rigid chains. The elastic modulus as a function of polymer volume fraction is determined for two cases : (a) the chains are rigidly crosslinked to each other i.e. the network may only deform by bending the chains (energetic network) and, (b) the chains are allowed to rotate freely about the crosslink points so that there is entropy associated with the crosslinks. A crossover concentration between the two behaviours $\phi_{\mathrm{c}}$ is defined. The reinforcement of a rigid network, by introducing a small concentration $c_{P}$ of straight rod-like elements of length $P$ (much greater than the mesh size of the lattice) is found to increase the modulus by a factor, $G_{P} \propto c_{P} P$. Finally we discuss how rigid networks may swell, and "de-swell» if the solvent quality is changed and calculate a possible form of the swelling ratio, as a function of the length of the network chains. The system is found to be unstable for a swelling ratio greater than $v_{2 \text { eq }}=(5 / 4)^{3}$.
\end{abstract}

\section{Introduction.}

Rigid networks are present in many guises in nature, for instance, silica gels, and the fractal structures arising from irreversible aggregation are often rigid. Polymers which are naturally stiff, such as helical molecules (PBLG), polydiacetylene, kappacarrageenan, polyamides and

(*) Present Address : Cavendish Laboratory, Cambridge CB3 OHE, U.K. 
peptides, can aggregate to form networks. We will model these networks as stiff elements joined at crosslink points. The elasticity of the network will depend upon the stiffness of the elements and the degree of mobility of the chains at each crosslink point.

It is important to quantify the rigidity of the chain. Even classical flexible chains, entirely dominated by the entropy associated with their myriad of configurations, are stiff on a short enough length scale (that of individual bonds). Such chains behave, for short enough deformations, as springs with spring constants which scale with the temperature $T$, i.e. $k \propto \frac{T}{N}$ (where $N$ is the degree of polymerisation of the chain). The scaling with $T$ indicates that the deformation is restricting the number of chain configurations, causing the entropy to drop. Our chain will be rigid in the sense of the spring constant being independent of $T$, the distortions are paid for with bend energy. We will first review a model of Ball and Kantor [1] which quantifies what level of stiffness is required before the network is energetic rather than entropic. Other discussions dealing with the cross-over from a rigid chain to a flexible one may be found in references [2] and [3]. Then we will go on to calculate the spring constant of a frozen chain with an arbitrary shape in the limit of small deformations. From the assumptions of affine deformation and rigidity of the crosslinks, will emerge laws governing the scaling of the elastic modulus with volume fraction of the chains in the network.

Even in the case where the network is built from rigid chains, its elasticity may be dominated by entropic rather than energetic effects. This is possible if the elements can rotate freely around the crosslink points and the topological constraints are "soft " enough. This problem has been considered by Edwards et al. [4]. The scaling of the elastic modulus with the volume fraction can be different from the system with rigid crosslinks. These two different behaviours can be simultaneously displayed and we will discuss the conditions under which each of them can be observed. We will mainly concern ourselves with modelling networks with rigid crosslinks, so there is no entropy associated with the system due to the free rotation of stiff elements about hinges, although we will discuss entropic contributions to the modulus in section 4.

Reinforcement of the rigid gels can be achieved by the introduction of extra chains in the network. We will calculate the increase of the elastic modulus of the network, as a function of the added concentration of rigid rods and their size.

We shall conclude with a calculation of the swelling and "de-swelling " of rigid networks with changing solvent quality. Again molecular weight between crosslink points is important.

\section{Chain rigidity.}

In order to arrive at a criterion for the rigid chain limit, in terms of polymer chain parameters, Ball and Kantor [1] consider a chain in the form of a frozen random walk. See figure 1. The chain is represented by a walk of $N$ steps, each of length $a$. The equilibrium angle for the joint between the $i$-th step and the $(i+1)$-th step is $\phi_{i}$, where $\phi_{i}$ may take any value between 0 and $2 \pi$. The random walk is frozen in the sense that an energy, $U=B\left(\phi_{i}-\theta_{i}\right)^{2} / 2$ is required to go from the equilibrium angle $\phi_{i}$ to $\theta_{i}$. B is the elastic force constant. If we then consider applying a force $\mathbf{F}$ to the ends of the chain the distortion can be found, enabling the modulus to then be determined. In this paper we are essentially only interested in two particular limiting values of the above quantities. Let us consider the limit for which $N k T / B \gg 1$, i.e. this is the high temperature, low $B / N$ region. The distortion $X$ of the chain in this case is found to be

$$
X=\frac{N a^{2} F}{k T}
$$




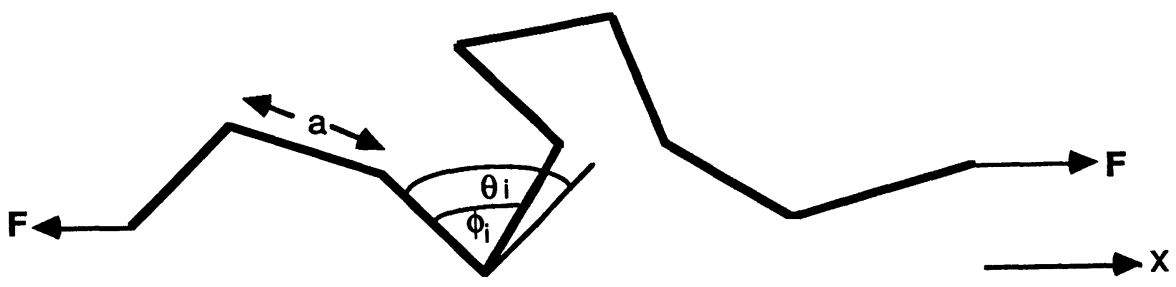

Fig. 1. - A chain in the form of a frozen random walk of $N$ steps each of length $a$. The angles between consecutive steps are only allowed to change by small amounts when the force $\mathbf{F}$ is applied to the ends of the chain.

which leads to the Gaussian chain result for the modulus and is to be expected at high temperatures. Now taking the high $B / N$, low $T$ limit i.e. when $N k T / B \ll 1$, the distortion is

$$
X=\frac{F(a N)^{2}}{4 B} .
$$

which has a dependence on $B$ but no $T$ dependence, unlike the Gaussian chain result. It should be noted that an alternative way of discussing the two limits is in terms of the thermal persistence length of the molecular chain $\ell_{\mathrm{p}}=B a / k T$. The high temperature limit corresponds to $\ell_{\mathrm{p}} \ll L$ ( $L$ is the contour length of the chain), so that the chain is flexible and we not surprisingly obtain the Gaussian chain result. The low temperature limit, however corresponds to $\ell_{\mathrm{p}} \gg L$ and so the chain is rigid.

In the following discussion we shall limit ourselves to networks composed of elements which are energetic rather than entropic in their elasticity. These elements, e.g. the molecular chains, may be conceived as frozen chains with some fractal dimension $1 / \nu$ connecting the size $R$ to the mass distribution : $R=N^{\nu} a$, where $a$ is a length of the order of the monomer size. As an example when $\nu=1$ we have a network of rod-like chains.

\section{Elastic response of rigid chains.}

3.1 BENDING A RIGID ROD. - The deformation of objects with large aspect ratios, for instance rods or frozen chains with a fractal dimension close to unity, is achieved most easily for a given applied force by bending rather than stretching or compressing. This notion is the basis for the elasticity of cellular structures (Ashby and Gibson [5]) and has been applied to tenuous fractal elasticity (Kantor and Webman [6]).

Applying a force $\mathbf{F}$ transversely to the end of a single rod produces a deflection $X$ which scales as $L^{3}$ for a fixed $\mathbf{F}$. See figure 2 . In the limit of small deformations the rod behaves, as expected, like a spring, with a spring constant of

$$
k=\text { const. } \frac{E t^{4}}{L^{3}},
$$

where $E$ is the rod material modulus, $t$ the cross-sectional dimension, and the constant depends on boundary conditions and on the shape of the cross-section. We ignore details since we are interested only in scaling. In the case of a rod-like molecular chain, it is not meaningful to talk in terms of the modulus and the cross-section of the "rod material». Instead we consider the quantity $B a$, the bend constant of the chain (where $B a=E t^{4}$ ), which is directly proportional to the persistence length of the chain. We will calculate the magnitude of this quantity for a given system in section 4 . 


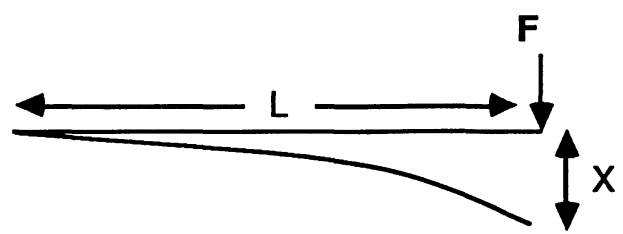

Fig. 2. - The rod bends when a force $\mathbf{F}$ is applied, producing a deflection $X$.

3.2 BENDING A FROZEN CHAIN WITH AN ARBITRARY SHAPE. - The assumption that the polymer chains are straight rods may be severe, and it is worthwhile looking in more detail at the conformation of the chains. A chain which is typical of the stiff synthetic systems we have in mind is given in figure 3. This has many benzene rings joined together, and gives rise to a structure, which is not linear see figure 4 , but has an overall linearity. Stricter linearity can be obtained by using molecules with linkages of the type shown in figure 5 which are discussed in reference [7].

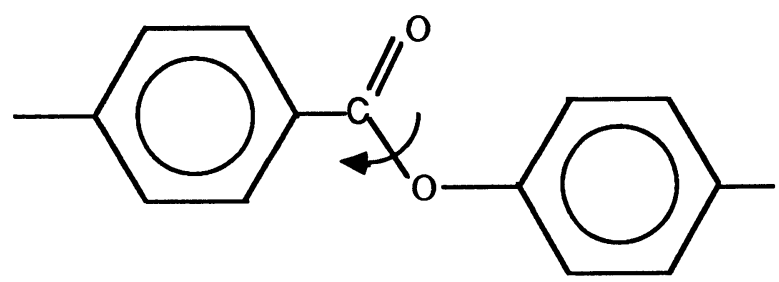

Fig. 3. - Part of a molecular chain which will deform by rotation about the bonds.

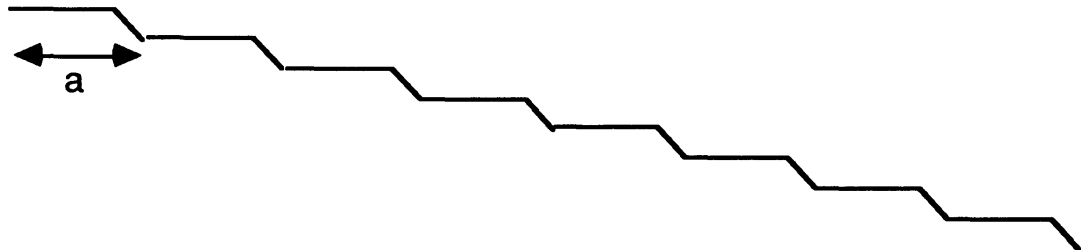

Fig. 4. - A schematic diagram of a molecular chain formed from the type of molecules in figure 3.

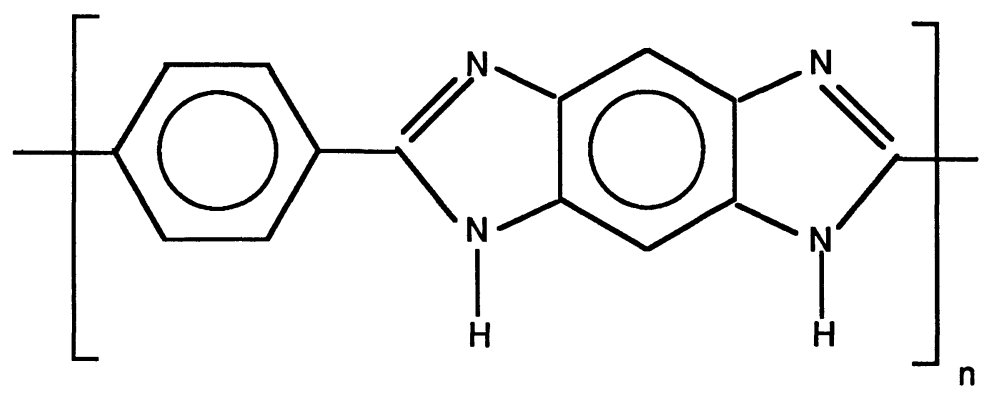

Fig. 5. - Linear chains can be formed using the type of molecules shown above, as in Husman and Helmimiak [7]. 
As we have discussed, if the force constant preventing deformation is quite large, we have an energetic network. It may be the case though that in the real system, over long time periods, i.e. long compared with the time for the modulus to be measured, the bonds may flip around and the structure exhibit behaviour more characteristic of flexible chains. The chains may then have entropic contributions to their elastic energy and have a temperature dependent modulus, for long time measurements.

Returning, to short time modulus measurements, where large changes in the shape of the chain are not allowed it is possible that the chain has a rigid, but random structure. For chains which are not straight we consider a more general case i.e. that of a rigid but arbitrary shaped chain. This problem has been considered before by Kantor and Webman [6] for fractal structures, Ball and Kantor [1] and Brown [8]. A simple form of the type of calculation is given below. Consider the arbitrary shaped element in figure 6 with forces applied to each end (the ends being the crosslink points that mechanically couple the chain to the network). The torque on the $i$-th element is $B \Delta \theta_{i}=F r_{i}^{\perp}$ where $\Delta \theta_{i}$ is the angle through which the $i$-th element of the chain bends through as it is strained and $r_{i}^{\perp}$ is the perpendicular distance from the point of application of the force to the $i$-th element of the chain. The change in length in the $x$-direction is $\Delta R=\sum_{i} \Delta r_{i}^{\|}$where $\Delta r_{i}^{\|}$is the change in the length of the $i$-th element in the direction parallel to the $x$-direction.

$$
\begin{gathered}
\Delta R=\sum_{i} \Delta \theta_{i} r_{i}^{\perp}=\sum_{i} F r_{i}^{\perp 2} / B . \\
\Delta R=\frac{F N}{B}\left\langle R^{2}\right\rangle .
\end{gathered}
$$

The arbitrarily shaped chain behaves, under small deformations, as a spring with a spring constant

$$
k=\frac{B}{N\left\langle R^{2}\right\rangle} .
$$

It can be seen that for $\nu=1 / 2$ this gives equation (2), so that equation (2) is just the special case of equation (6), where the chain has the configuration of a random walk. In the limit of the fractal dimension being one, we recover the rod spring constant of equation (3) (using $B a=E t^{4}$ ). For fractal dimensions higher than one, the equivalent spring rigidity is smaller by

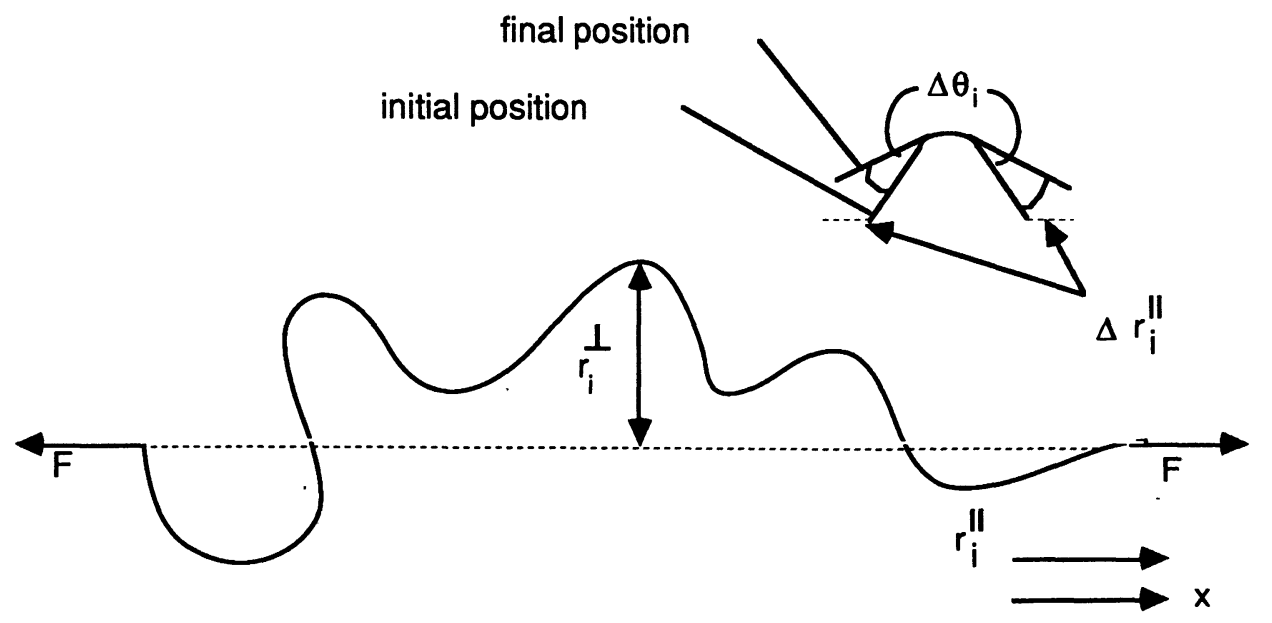

Fig. 6. - A randomly shaped rigid chain, which may deform by bending when a force is applied to its ends. 
a factor of $R / N \sim N^{\nu-1}$. As in the preceding paragraph the calculation ignores the extensibility of the rigid elements of the chain, since this leads to a less compliant elastic response.

\section{Elasticity of a network of rigid chains.}

4.1 FROZEN CROSSLINKS. - We first consider systems where there is a fixed number of chemical crosslinks which are permanent and rigid i.e. they are not freely hinged. We now want to determine the modulus of a network of this type. A qualitative argument to derive the shear modulus is given first and a more detailed and more general argument is given later.

If we apply an external force to the network it will be transmitted by the rigid system. We make the assumption that the system deforms affinely down to a length-scale $\sim O(R)$, the distance between crosslinks. Although it is not clear that this assumption should hold for rigid chains for the types of deformations that we are considering, we use it here as an attempt to obtain a simple, first order model of the processes involved when rigid networks deform.

If there is a force $F$ acting on each chain, and a total of $n$ chains in a volume $R^{3}$, then the stress carried is $\sigma=n F / R^{2}$. Because of the affine assumption the strain of the network will be given by $\varepsilon=X / R$. Young's modulus $G_{\mathrm{r}}$ for small deformations is then given by,

$$
G_{\mathrm{r}}=\frac{\sigma}{\varepsilon}=\frac{B n}{N R^{3}}
$$

We define the volume fraction $\phi$ of polymer in our system as follows. If there are $n$ chains in a volume $R^{3}$, each of volume $\sim N a^{3}$ (where $a$ is the typical monomer dimension and the crosssection $t$ is taken to be the monomer length $a$ ) then $\phi$ is

$$
\phi \sim \frac{n N a^{3}}{R^{3}} \sim n N^{1-3 \nu} .
$$

the later relation relying on $R \sim N^{\nu} a$. In our modelling we assume that $n$ is small so that the effect of entanglements can be ignored. The volume fraction may be varied by holding $n$ fixed and allowing $N$ to vary, or by fixing $N$ and allowing $n$ to change. The later of these can be achieved by controlling the functionality of the crosslinks. If $N$ is constant then we obtain the following scaling of the modulus with concentration.

$$
G_{\mathrm{r}}=\frac{B \phi}{a^{3} N^{2}} .
$$

If we fix $n$ and allow $N$ to vary then we obtain a different scaling of modulus with concentration, as given below

$$
G_{\mathrm{r}}=\frac{n B}{a^{3}}\left(\frac{\phi}{n}\right)^{\left(\frac{3 \nu+1}{3 \nu-1}\right)} .
$$

There exist more general arguments to calculate the stress in a network which do not rely on defining an area and a volume for each molecular chain, see Doi and Edwards [9]. The stress across a given area, see figure 7 is then given by the following argument.

$$
\sigma_{\alpha \beta}=\frac{1}{V} \sum_{m, n} F_{m n}^{\alpha}\left(R_{m}^{\beta}-R_{n}^{\beta}\right)
$$

where $\mathbf{R}_{m}$ is the position of the $m$-th junction and $\mathbf{F}_{m n}$ is the force exerted by the point $n$ on the point $m$ (in this case via the connecting chain). Although the application of equation (11) does 

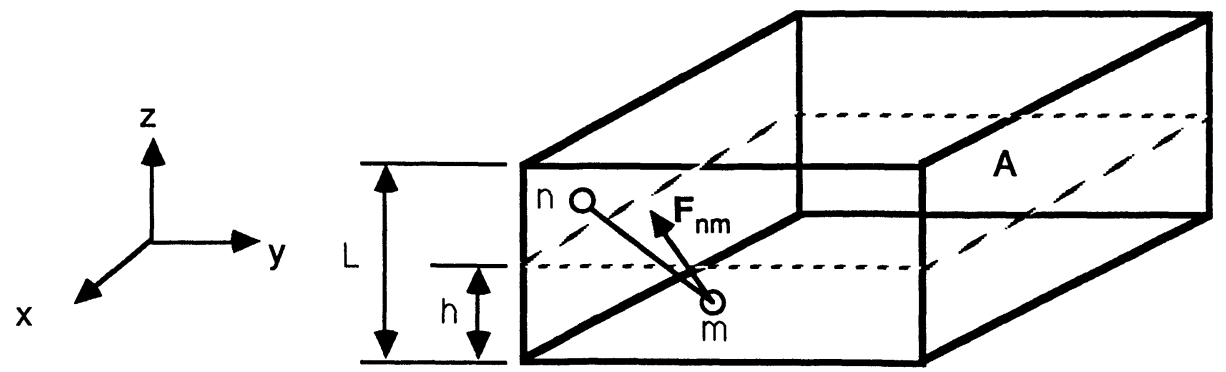

Fig. 7. - Definition of the stress tensor $\sigma_{\alpha \beta}$. The component of the stress $\sigma_{\alpha z}$, is the $\alpha$ component of the force (per area) that the material above the plane of area $A$ and $a$ distance $h$ from the origin exerts on the material below the plane.

not allow us to obtain a precise result for the modulus, it is worth following through the argument since the underlying assumptions are drawn sharply into focus.

Considering figure 8 there are three rods exerting forces and torques at $m$. The analysis is extremely involved and so we introduce some simplifying assumptions. These involve : (a) Taking only the reaction, $-\mathbf{F}_{m n}$ to evaluate the distortions of strut $m n$. This represents the action of the counter-couples at $n$ by clamping and vice versa for the couples at $m$. (b) Assuming all of the $\mathbf{F}_{m n}$ is effective in bending $m n$. (c) Assuming that the deformation $\Delta \mathbf{R}_{m n}$ is affine. Then if we consider the deformation of one rod, we obtain

$$
\Delta \mathbf{R}_{m n}=\mathbf{R}_{m n}-\mathbf{R}_{m n}^{0}=(\Lambda-\mathbf{1}) \mathbf{R}_{m n}^{0} .
$$

where $(\boldsymbol{\Lambda}-\mathbf{1})$ is the strain and following (b) above we get

$$
\Delta \mathbf{R}_{m n}=\frac{\mathbf{F}_{m n} L^{3}}{E t^{4}} .
$$

If we impose a uniaxial strain $\lambda_{z z}$, inserting the above into equation (11) we obtain

$$
\sigma_{z z}=\frac{1}{V} \frac{E t^{4}}{L^{3}} \sum_{\text {strands }} \lambda_{z z}\left(\lambda_{z z}-1\right)\left(R_{m n, z}^{0}\right)^{2} .
$$

Averaging this using, $R_{m n, z}^{0}=L \cos \theta$ and $\left\langle\cos ^{2} \theta\right\rangle=1 / 3$ (which assumes an isotropic chain distribution) yields

$$
\sigma_{z z}=\frac{E t^{4} N_{\mathrm{s}}}{3 L V}\left(\lambda_{z z}-1\right) \lambda_{z z} .
$$
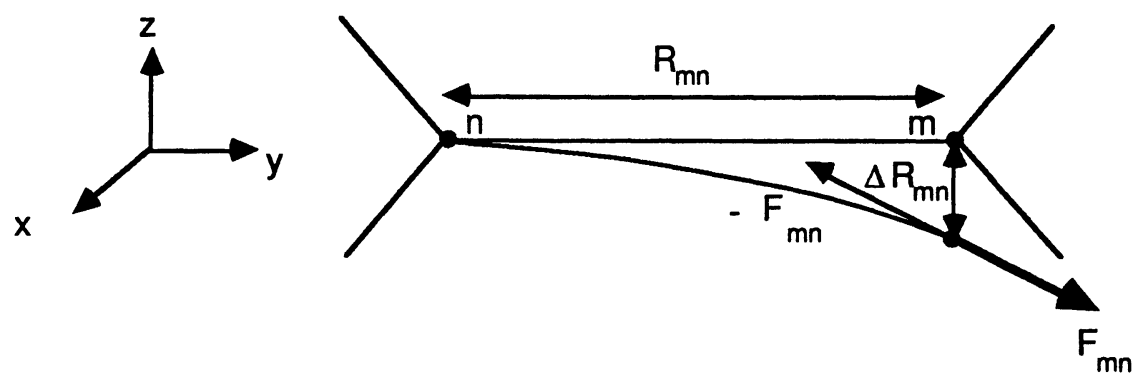

Fig. 8. - Derivation of the stress tensor $\sigma_{z z}$. The component of $\mathbf{F}_{m n}$ in the $z$-direction causes a change in the $z$-component of $\mathbf{R}_{m n}$. 
For the modulus $\partial \sigma_{z z} / \partial \varepsilon_{z z}$ with $\varepsilon_{z z}=\left(\lambda_{z z}-1\right)$ we get a result differing only by unimportant factors from equation (7). For a straight chain $B a=E t^{4}$ and in equation (15) $N_{\mathrm{s}}$ is defined as the number of chains in a volume $V$.

Returning to equation (10), we can choose to follow the scaling properties by varying $\phi$, the volume fraction of the polymer, by allowing $N$ to vary, at fixed $n$. When the chains are straight (fractal dimension $1 / \nu=1$ ) the elastic modulus scales as the second power of the polymer volume fraction, as is found for a wide class of cellular structures as shown in [5].

$$
G_{\mathrm{r}} \sim \phi^{2} \quad \nu=1 .
$$

This is in good agreement with the experimental results of Rinaudo et al. on the elastic modulus of kappa-carrageenan gel which gives a power law dependence of $G \sim \phi^{2 \pm 0.01}$ as discussed in [10]. The chains of this gel are believed to have a very large persistence length and, indeed, electron micrographs show a structure which could be described as an entanglement of almost straight chains see reference [11].

Another system which could provide a convincing test of our predictions is the silica gel system. These gels have been characterized by several experimental groups. By varying the $\mathrm{pH}$ of the reaction bath which produces the gel it is possible to modify, at least to some extent, its fractal dimension. Schaefer et al. [12] report fractal dimensions obtained by SAXS varying from 1.7 to 2.3. We are not aware of any systematic measurement of the elastic modulus of the gel as a function of its fractal dimension but results reported by Dumas et al. [13] give a power law dependence of $G \sim \phi^{5 \pm 0.5}$, consistent with the fractal dimensions of order of two reported by Schaefer.

The value of $B$ in the above equation may be estimated for a particular system, that of figure 3. It can then be used to determine the order of magnitude of the modulus expected for such systems, and a comparison be made with typical experimental data. It is assumed that for a molecular chain such as figure 3 , the bend is achieved by rotation about molecular bonds. Rotation about the bond shown causes the chain to bend out of the plane of the paper. This is shown in the more schematic diagram figure 9. This mechanism is different in detail from that of figure 1. We assume coplanarity of the two successive benzene rings and their connection is a quadratic minimum of the energy, $U=C \omega^{2} / 2$, where $\omega$ is the rotation about the $\mathrm{CD}$ axis (systems not obeying this type of potential are beyond the scope of this paper). The effect is to move end $E$ from coplanar position $E_{1}$ to the elevated position $E_{2}$. For small $\omega$ the displacement is perpendicular to the plane and is given by $\sim \omega \sin \gamma \mathrm{DE}$, and the effective angle bent through is $\alpha \sim \omega \sin \gamma \mathrm{DE} / \mathrm{CE}$. The rate of change of angle with distance moved along the chain is $\alpha / C E$, and the bend constant is $B a=B$. CE. The energy for bending the chain is then given by $U=B(\mathrm{CE})^{2}(\alpha / \mathrm{CE})^{2} / 2$. The expression for the bend constant being $B a=C$. CE $(\mathrm{CE} / \mathrm{DE} \sin \gamma)^{2}$. Then by using data giving the variation of $U$ with $\omega$ we can calculate $C$ and thus $B a$. Obviously the exact value of $B a$ depends on the geometry of particular molecules, and so we only give an order of magnitude estimate for a typical system, that of figure 3. We thank Dr. A. H. Windle for supplying the potential data. Assuming that $\mathrm{DE} / \mathrm{CE} \sim 1 / 4, \sin \gamma=\sqrt{3} / 2$ and that $\mathrm{CE}=a \sim 10^{-9} \mathrm{~m}$ we obtain $B a \sim 10^{-27} \mathrm{Jm}$. Then using $N \sim 10$, and $\phi \sim 10^{-1}$ we obtain a value for the elastic modulus of $G \sim 10^{6} \mathrm{~Pa}$. In the work of Aharoni and Edwards [14] the modulus is found to be of the order $10^{6} \mathrm{~Pa}$, and over the range of temperature investigated, it is independent of temperature, which fits the energetic network model.

4.2 REINFORCEMENT OF THE GEL. - It may be of some practical interest to consider a system into which there has been introduced a small concentration $c_{P}$ of chains with a size much larger than the average mesh size of the network. For the sake of simplicity we only 


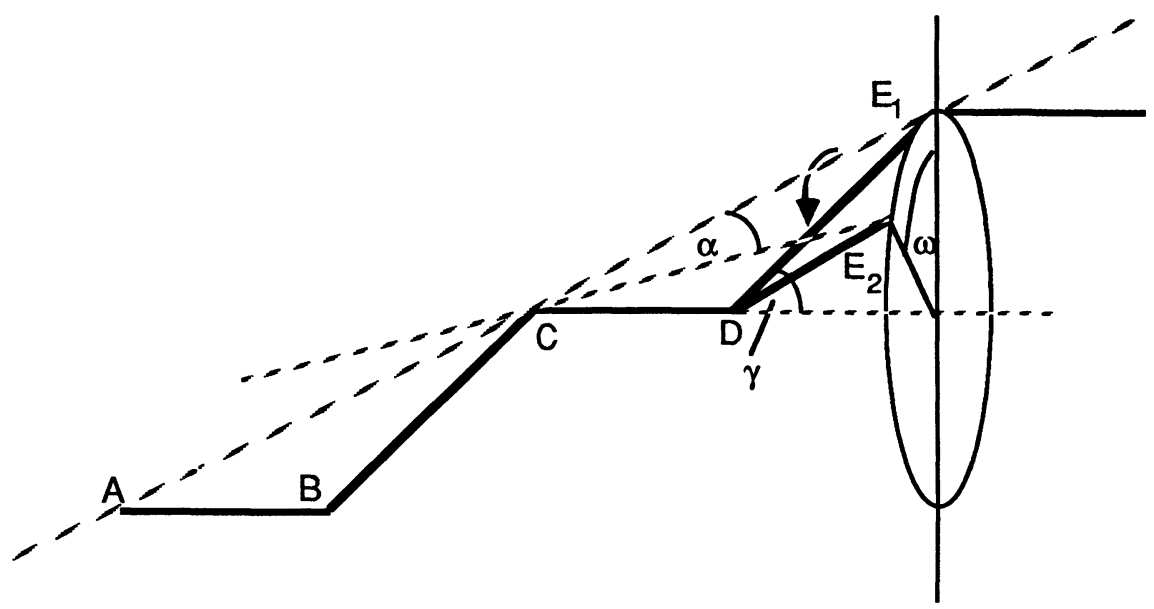

Fig. 9. - Diagram to show how the bend of a molecular chain may be achieved by rotations about bonds. The segments $B C, C D$ and $D E$ are part of a chain. When $E$ is in position $E_{1}$ there is a linearity a long $\mathrm{AE}_{1}$. If the segment $\mathrm{DE}$ rotates about the axis $\mathrm{CD}$, through an angle $\omega$ out of the plane of the paper so that the end moves from position $E_{1}$ to position $E_{2}$ then the line connecting $C$ and $E$ is no longer parallel to $\mathrm{AE}_{1}$ and has rotated through an angle $\alpha$ from the direction of $\mathrm{AE}_{1}$. Therefore successive rotations along the molecular chain give rise to bending of the chain.

discuss the case of straight chains (of length $P$ ) being introduced into a network of rods of size $L(L \ll P)$. When a force is applied to the network there are two types of chain-chain contacts which transmit the stress. The first ones are the permanent crosslink points that have been discussed in the preceding section i.e. chemical crosslinks consisting of covalent bonds. In addition to these ones there will be rod-rod contact points which are formed when a $P$ rod meets other $L$ rods along its length, we will call these contact points extra links. They are extra in the sense that as the network is deformed the rods will form more of these contacts. This type of contact has been considered before in relation to rigid networks by Doi and Kuzuu [15]. In [15] the only type of links in the system are physical ones, formed by rod-rod contacts occurring during deformation. In such a network it is found that the elasticity is highly nonlinear. In the network which we discuss this non-linear elasticity is not present, at a first level of approximation, since the stress carried by the extra links is transmitted via the network of fixed crosslinks. Non-linearity would only occur at higher concentrations of added $P$ rods.

The extra links increase the free energy of the system and; following [15] we write this extra free energy per unit volume as

$$
A_{P}=c_{P} B a \int_{0}^{P}\left\langle\left[\frac{\mathrm{d}^{2} \xi}{\mathrm{d} x^{2}}\right]^{2}\right\rangle \mathrm{d} x
$$

where $\xi(x)$ is the amount of deflection imposed to one rod by the deformation of the network. A rough estimate of the extra energy can be obtained from $\langle\mathrm{d} \xi\rangle$, the averaged deformation imposed on the $P$ rods, and $\left\langle\mathrm{d} x^{2}\right\rangle$, the squared average distance between two points carrying the stress. The assumption of affine deformation leads to

$$
\langle\mathrm{d} \xi\rangle \sim \varepsilon L ;\left\langle\mathrm{d} x^{2}\right\rangle \sim L^{2}
$$

where $\varepsilon=(\lambda-1)$. The introduction of the $P$ chains $(P \gg L)$ thus reinforces the elastic 
modulus of the gel by a factor which increases linearly with concentration and with the size of the extra rods, giving a contribution to the modulus of

$$
G_{P} \sim c_{P} \frac{B a P}{L^{2}} .
$$

4.3 FREELY HINGED NETWORK. - We consider in this paragraph a system which is made from chains of average size $R$ meeting at permanent crosslink points of functionality $z$. The chains are freely hinged in the sense that if one isolates from the network the $z$ chains meeting at some crosslink point, there will be no constraint on the angles between the chains. Thus, in such a network, the constraints acting on the crosslinks are only derived from the topology of the system. Other work on freely hinged systems has been done by Thorpe and Phillips [16] and also Edwards et al. in [4]. We expect that a very high functionality will completely freeze the degrees of freedom of the system. This problem has been considered before by Vilgis et al. in reference [17]. In order to estimate the critical functionality which would freeze the angles, we describe the system as an ensemble of $N$ points - the crosslink points - each of them connected to $z$ neighbours. The description of the dynamics of these $N$ points in a $d$ dimensional space needs $N d$ coordinates. There are, in a very large system, $N z / 2$ constraints of the type $\left|r_{i}-r_{j}\right|=L_{i j}$. The total number of degrees of freedom per crosslink point is then

$$
\theta=d-\frac{z}{2}
$$

If one wants to keep some mobility in a three dimensional system, the functionality needs to be less than six. For $z=4$ there will be a degree of freedom per crosslink point. We now assume that the functionality is low enough in order to keep some internal mobility in the system. At non-zero temperature, the thermal fluctuations of the system determine its susceptibility

$$
\chi=\frac{\left\langle Y^{2}\right\rangle}{k_{\mathrm{B}} T}
$$

where $Y$ is the average vector joining the two points which represent the extreme positions of the end of the chain as the chain undergoes thermal fluctuations. Let $\alpha$ be the angle formed by two chains meeting at a crosslink point. The fluctuations in the positions of the end of any particular chain depend upon the fluctuations $\delta \alpha$ of the angle between the two chains about its average value of $\alpha$. Therefore since $R$ is the end to end distance of the chain we obtain the following equation for $\left\langle Y^{2}\right\rangle$

$$
\left\langle Y^{2}\right\rangle=R^{2}\left(\delta \alpha^{2}\right) .
$$

The susceptibility $\chi$ is the inverse of the force constant $k$. The elastic modulus can thus be written as a function of the angle fluctuations $\left\langle\delta \alpha^{2}\right\rangle$ and the distance between crosslinks $R$ :

$$
G_{\mathrm{e}}=\frac{n k_{\mathrm{B}} T}{R^{3}\left\langle\delta \alpha^{2}\right\rangle} .
$$

The exact value of $\left\langle\delta \alpha^{2}\right\rangle$ depends in a very complicated way on the topology of the network and will remain as an unknown parameter. From equation (8) we get the scaling of the elastic modulus with the volume fraction of the polymer for constant $n$ to be

$$
G_{\mathrm{e}} \sim \frac{n k_{\mathrm{B}} T}{\left\langle\delta \alpha^{2}\right\rangle a^{3}}\left(\frac{\phi}{n}\right)^{3 \nu / 3 \nu-1} .
$$


The elasticity of this entropic network of rigid chains has a very different scaling from the system with frozen crosslinks (compare with Eq. (10)) :

$$
\begin{cases}\nu=1 & G_{\mathrm{e}} \sim \phi^{3 / 2} \\ \nu=\frac{1}{2} & G_{\mathrm{e}} \sim \phi^{3} .\end{cases}
$$

The entropic and enthalpic type of elastic behaviours can be simultaneously present in actual systems, depending on the rigidity of the chains and on the magnitude of the fluctuations of the angles. At a macroscopic level there is an imposed stress $\sigma$ and the two different contributions to the elastic modulus act in parallel. The resulting modulus $G$ is then given by

$$
G^{-1}=G_{\mathrm{r}}^{-1}+G_{\mathrm{e}}^{-1}
$$

Comparing the two contributions it is natural to define the crossover concentration $\phi_{\mathrm{c}}$ that can be written as

$$
\phi_{\mathrm{c}} \simeq n\left[\frac{k_{\mathrm{B}} T}{B\left\langle\delta \alpha^{2}\right\rangle}\right]^{2}
$$

for straight chains $(\nu=1)$. For volume fractions larger than $\phi_{\mathrm{c}}$ the elastic behaviour has an entropic origin and the elastic modulus follows the scaling of equation (24). For volume fractions smaller than $\phi_{c}$ the deformation of the network is accomplished by the bending of the rods leading to the scaling of equation (10). In practice a large rigidity of the chains (large $B$ ) and a large thermal fluctuation of the angles (large $\left\langle\delta \alpha^{2}\right\rangle$ ) lead to a very small crossover concentration and the displayed behaviour is entropic.

\section{Swelling of rigid networks}

When networks come into contact with solvent three main effects can exist. First there is the preference of the chains for each other or for solvent, which following Flory [18] and Huggins [19] is conventionally expressed in terms of mean field theory as giving an energy change per monomer site of $k T \chi \phi(1-\phi)$ when mixing occurs. $\chi$ is the solvent quality and is negative for solvent absorption into the network to be favoured, and positive for expulsion. Secondly, if solvent is imbibed, the chains must extend so reducing their entropy, and there will be an elastic restoring force, opposing extension. In the networks described in this paper, then at least at short times, these forces will be energetic. At long times it is possible that the chains display entropic behaviour due to bond flips occurring. The third contribution is an entropy of mixing when solvent is absorbed. This is only present when the chains are flexible, that is in the long time entropic limit, where configurational changes are allowed. In the rigid system no such entropy of mixing term exists. In the general case these three effects compete with each other and the system reaches swelling equilibrium when the total energy of the system is a minimum with respect to the amount of solvent taken up.

In this section we first want to consider the case of a rigid network made of energetic chains, and to ask the question of how the network swells. The swelling process brings into focus several problems still to be resolved in the modelling of rigid systems. - How much does rigid chain overlap effect classical swelling, to what extent is Doi and Kuzuu treatment required ? Over what length scale is the chain deformation affine with the bulk ? A large amount of work has been done by Bastide $e t$ al. on the de- swelling of gels and their deformation mechanism, which can be found in reference [20]. 
In rigid systems the entropy of mixing term discussed above vanishes (because no configurational changes are allowed to occur), and therefore we can only consider the $k T \chi \phi(1-\phi)$ term to be driving the swelling. Thus swelling will only occur if $\chi$ is negative so that the energy can be lowered.

We make the assumption in our model that for the swelling process the network is phantom i.e. the chains may pass through each other. This is admittedly a dramatic simplification and ignores such difficulties as log-jams. On the other hand we assume contacts between chains and solvent are described by $k T \chi \phi(1-\phi)$, which implies that the number of rod-rod contacts scale as $\phi^{2}$. We shall use it however to give a first approximation to the swelling mechanism. The total free energy of mixing of the network is written as

$$
F_{\text {tot }}=F_{\text {mix }}+F_{\text {el }} \text {. }
$$

The elastic free energy is given by

$$
\begin{gathered}
F_{\mathrm{el}}=n_{\mathrm{s}} \int_{\mathrm{d}} F \mathrm{~d} R \\
\Rightarrow F_{\mathrm{el}}=\frac{n_{\mathrm{s}} B}{2 N}(\lambda-1)^{2}
\end{gathered}
$$

where $\lambda R_{0}=R$ and $R_{0}$ is the unstretched value of the end to end chain length, and $n_{\mathrm{s}}$ is the total number of chains. Also, for isotropic swelling the extension ratio $\lambda$ is

$$
\lambda^{3}=\frac{V}{V_{0}}=\frac{1}{v_{2}}
$$

i.e. the quantity $v_{2}$ is defined as the ratio of the initial and final volumes of the network. Therefore, if $n_{1}$ solvent monomers mix with a fixed number, $n_{2}$ of polymer monomers in the network, then $v_{2}=\left(n_{2}+n_{1}^{0}\right) /\left(n_{2}+n_{1}\right)$ where $n_{1}^{0}$ is the initial number of solvent monomers. The volume fraction of polymer is then $\phi=n_{2} /\left(n_{2}+n_{1}\right)$, and the initial value is $\phi_{0}=n_{2} /\left(n_{2}+n_{1}^{0}\right)$, so that $v_{2}=\phi / \phi_{0}$. The total solvation energy when $n_{1}$ monomers of solvent are absorbed is given by

$$
F_{\text {mix }}=k T \chi n_{1} v_{2} \phi_{0}
$$

In order to find the equilibrium swelling point the total free energy $F_{\text {tot }}$ must be minimised with respect to $n_{1}$. This gives,

$$
0=v_{2 \mathrm{eq}}^{2} \chi \phi_{0}^{2}+\frac{B n_{\mathrm{s}} \phi_{0}\left(v_{2 \mathrm{eq}}^{1 / 3}-v_{2 \mathrm{eq}}^{2 / 3}\right)}{3 N k T n_{2}} .
$$

It can be seen that if we consider the small swelling limit, so that $v_{2 \text { eq }}=1-\delta$ where $\delta \ll 1$ then we obtain

$$
\chi=\left(2 \chi-\frac{B}{9 N^{2} k T \phi_{0}}\right) \delta
$$

which for $B / 9 N^{2} k T \phi_{0} \gg 2 \chi$ leads to

$$
v_{2 \mathrm{eq}} \simeq\left(1+\frac{\chi^{9} N^{2} k T \phi_{0}}{B}\right) .
$$


If we take the large swelling limit i.e. $v_{2}^{1 / 3} \gg v_{2 \text { eq }}^{2 / 3}$ then we arrive at the result

$$
v_{2 \mathrm{eq}}^{5 / 3} \simeq \frac{B}{3 N^{2} k T|\chi| \phi_{0}}
$$

It is interesting at this point to compare these results with the corresponding ones for a flexible network. For a classical Gaussian chain the elastic free energy is given by

$$
F_{\mathrm{el}}=\frac{3 k T n_{\mathrm{s}}}{2}\left(\lambda^{2}-1\right) \text {. }
$$

The energy of mixing of an entropic network with solvent contains both an entropic mixing term and an energy of solvation due to solvent interactions. For the entropic network and taking $\phi_{0}=1$, we have

$$
F_{\text {mix }}=k T n_{1} \ln v_{1}+k T \chi n_{1} v_{2} .
$$

For this type of network the equilibrium condition can be determined and is found to be,

$$
0=v_{2 \mathrm{eq}}+\ln v_{1 \mathrm{eq}}+\chi v_{2 \mathrm{eq}}^{2}+\frac{n_{\mathrm{s}}}{\lambda n_{2}} .
$$

In the large swelling limit i.e. $v_{2 \text { eq }} \ll 1$ we obtain,

$$
\begin{aligned}
& v_{2 \mathrm{eq}}=\left(\frac{n_{\mathrm{s}}}{n_{2}(\chi-1 / 2)}\right)^{3 / 5} \\
& v_{2 \mathrm{eq}}=\left(\frac{1}{N(\chi-1 / 2)}\right)^{3 / 5} .
\end{aligned}
$$

This shows a significant difference in the $N$ depedence of the swelling ratios for entropic networks and energetic networks.

We can also look at how the network may « de-swell », i.e. how solvent may be expelled, by using a poor solvent and allowing the network to decrease in volume. This type of behaviour will be seen in a rigid network only if $\chi>0$. We have already seen that swelling equilibrium is achieved when the osmotic pressure $\pi$ vanishes, and $\pi$ is given by

$$
\pi=-\frac{\partial F_{\text {tot }}}{\partial n_{1}}=-k T \chi v_{2}^{2} \phi_{0}^{2}-\frac{B \phi_{0}}{3 N^{2}}\left(v_{2}^{1 / 3}-v_{2}^{2 / 3}\right) .
$$

When $\chi>0$ it can be seen that there is a region in which the $-\chi v_{2}^{2}$ term dominates and the system will be unstable. This will occur when

$$
\frac{\partial \pi}{\partial v_{2}}<0
$$

and substituting in the condition at equilibrium (32) we obtain

$$
\left.\frac{\partial \pi}{\partial v_{2}}\right|_{v_{2 \mathrm{eq}}}=\frac{B \phi_{0}}{9 N^{2}}\left(5 v_{2 \mathrm{eq}}^{-2 / 3}-4 v_{2 \mathrm{eq}}^{-1 / 3}\right) \text {. }
$$

Therefore the model gives an instability for

$$
v_{2 \text { eq }}>(5 / 4)^{3} .
$$


Experimentally, the swelling of rigid networks has been performed by Aharoni [14] by changing the solvent quality. The swelling was performed over several months and factors of order two for the swelling ratio were observed. The experiments are done over long time periods in order to allow the solvent to enter into the networks slowly, otherwise the networks are destroyed by the process. We have mentioned already in section 3 that in addition to the energetic behaviour of these networks there could also be a different long time scale behaviour. This would occur because of bonds flipping over long time periods and cause configurational changes leading to entropic behaviour. These long time (slow) flips would give rise to a long time temperature dependent modulus, and in addition, because configurational changes can occur, the network could display a conventional swelling mechanism as described by FLory [18] and Huggins [19], if the time for swelling is greater than the time for bond flips to take place.

\section{Conclusion.}

We have presented a model for the deformation of a network made of rigid elements and found that, if the deformation is restricted to the bending of the chains then the scaling behaviour of the modulus with concentration under certain conditions depends on the fractal dimension of the chains. For straight rod-like chains this scaling being $\phi^{2}$, and for chains which are frozen random walks $\phi^{5}$. The other feature of this elastic modulus being that it is temperature independent, since it is derived from energetic rather than entropic effects.

We also consider an entropic contribution to the modulus for the case where the crosslinks are freely hinged and obtain a new set of power laws for the variation of modulus with concentration. The two moduli i.e. entropic and energetic, have different scaling with concentration and so we can define a crossover concentration $\phi_{\mathrm{c}}$ above which the entropic effects dominate the modulus, and it becomes temperature dependent.

If the network is reinforced by introducing a concentration $c_{p}$ of rods of size $P$ into the system, then for $P \gg L$ (where $L$ is the distance between crosslinks in the network) the modulus is found to be increased by a factor which is directly proportional to $c_{p}$ and $P$.

The swelling of rigid networks by imbibing solvent is a very complex problem and we have given only a first approximation as to the processes taking place. The equilibrium swelling ratio $v_{2 \text { eq }}$ is determined for the case where the crosslinks are rigid for a general fractal dimension $\nu$ of the chains, however it is difficult to visualise how the network can swell for $\nu=1$, and it is possible that the analysis breaks down before $\nu=1$. If the network "deswells" it is found that an instability exists when the ratio of initial to final volumes, $v_{2 \text { eq }}$ is greater than $(5 / 4)^{3}$. Although, there is only a limited amount of experimental data currently available for such systems, the results of [12] and [13] give the modulus versus concentration power law. The dependence of this power on the fractal dimension of the chains, agrees qualitatively with our predictions. In [14] the networks synthesised have a temperature independent modulus and to an order of magnitude the modulus measured agrees quantitatively with what is predicted for very similar systems by our model.

\section{Acknowledgements.}

The authors would like to thank M. Warner, J. F. Joanny, R. C. Ball, S. F. Edwards and S. M. Aharoni for many helpful contributions to this work. One of us (J. J.) is grateful for a C.A.S.E. award with Unilever, Colworth House and the Agricultural and Food Research Council. 


\section{References}

[1] BALl R. C. and Kantor Y., private communication.

[2] Landau L. D. and Liftshitz E. M., Statistical Physics Part 1 p. 396.

[3] Kantor Y. and Witten T. A., J. Phys. Lett. 45 (1984) L675-679.

[4] Boué F., Edwards S. F., Vilgis T. A., J. Phys. 49 (1988) 1635-1645.

[5] Ashby M. F. and Gibson L. J., Cellular Solids, Structure and Properties (Pergamon Press) 1988.

[6] Kantor Y. and Webman I., Phys. Rev. lett. 52 (1984) $\mathrm{N}^{\circ} 21$.

[7] Husman G., Helmimiak T., Adams W., Wiff D. and Benner C., Org. Coat. Plas. Chem. 40 797.

[8] Brown W. D., Ph. D. Thesis, Cambridge (1986).

[9] Dor M. and Edwards S. F., Theory of Polymer Dynamics (Oxford Press) 1986.

[10] RINAUDO, private communication.

[11] Rochas C., Ph. D. Thesis, Grenoble (1982).

[12] Shaefer D. W. and Keefer K. D., Phys. Rev. Lett. 56 (1986) N 20.

[13] Dumas J., Baza S. and Serughetti J., J. Mater. Sci. Lett. Vol. 5 (1986) 478.

[14] Aharoni S. M. and Edwards S. F., Macromolecules 22 (1989) 3361.

[15] DoI M. and Kuzuu N. Y., J. Polymer Sci.: Polymer Phys. Ed. 18 (1980) 409-419.

[16] See for example, Thorpe M. F. and Phillips J. C., Solid State Comm. 53 (1985) 699 and references therein ;

Phillips J. C., J. Non-Cryst. Sol. 34 (1979) 153 ;

ThORPe M. F. and Garboczi E. J., Phys. Rev. B 35 (1987) 8579.

[17] Vilgis T., BouE F. and Edwards S. F., Molecular Basis of Polymer Networks, Springer Proceedings in Physics 42, p. 170.

[18] FloRY P., Principles of Polymer Chemistry, Chapter 12 (Cornell University Press, Ithaca, New York) 1971.

[19] Huggins M., J. Phys. Chem. 46 (1942) 151 ;

Huggins M., Ann. N. Y. Acad. Sci. 41 (1942) 1 ;

Huggins M., J. Am. Chem. Soc. 64 (1942) 1712.

[20] Bastide J., Picot C. and Candau S., J. Macromol. Sci. Phys. B 19 (1981) 13 ;

Bastide J., Duplessix R., Picot C., Candau S., Macromolecules 17 (1984) 83. 